\title{
Development the Technique for the Preparation and Characterization of Reconstructed Human Epidermis (RHE)
}

\author{
Herlina B. Setijanti ${ }^{1,2}$, Eka Rusmawati ${ }^{1(\bowtie)}$, Rahmi Fitria ${ }^{1}$, \\ Tuty Erlina ${ }^{1}$, Rina Adriany ${ }^{1}$, and Murtiningsih ${ }^{1}$ \\ ${ }^{1}$ Research Centre for Drug and Food, National Agency Drug and Food Control \\ of Republic of Indonesia, Central Jakarta, Indonesia \\ bs_herlina_2002@yahoo.com \\ ${ }^{2}$ Faculty of Pharmacy and Science, HAMKA University of Republic \\ of Indonesia, South Jakarta, Indonesia
}

\begin{abstract}
Reconstructed Human Epidermis (RHE) is an artificial epidermis made in such a way that it resembles human skin, and can be used for the identification of irritant chemicals, especially for cosmetic and topical medicinal products. Currently the new RHE is produced by European and American countries, whose skin physiology is very different from Indonesia. Based on this, the Center for Research on Drugs and Food, NADFC of Republic of Indonesia took the initiative to develop the reconstruction of keratinocyte, melanocytes and fibroblasts cells into RHE adapted to the anatomical and physiological functions of the skin of Indonesians. RHE is made from an epidermal layer composed of keratinocyte and melanocytes cells that are reconstructed with a dermis layer composed of fibroblast and collagen cells. Keratinocyte, melanocytes and fibroblasts cells are cultured on suitable mediums by adding a suitable growth medium. To find out that RHE has been successfully reconstructed, measured percentage of cell life, made histology preparation to see the existence of cell nucleus, and conducted Immunohistochemical examination to see existence of integration (bond) between antigen. From the research results can be seen that keratinocyte cells grown on culture medium Keratinocyte SFM (IX) with rEGF supplements; melanocyte cells grown on Melanocyte 254 culture medium with HMGS supplementation; and fibroblast cells grown on Fibroblast M 106 culture medium with LSGS supplementation. The percentage of epidermal cell life grew well in the planting of $10 \times 10^{4}$ cells $/ \mathrm{mL}$ keratinocytes and $0.25 \times 10^{4}$ cells $/ \mathrm{mL}$ of melanocyte cells and survived until the $11^{\text {th }}$ day with live cell percentage of $93.45 \%$. In making preparation for histology with HE staining, there is a cell life in RHE tissue. Used Immunohistochemical (IHC) examination using cytokeratin 10 antibody marker to view physiological function of epidermal tissue.
\end{abstract}

Keywords: RHE $\cdot$ Keratinocytes cell $\cdot$ Melanocyte cell 


\section{Back Ground}

The wide use of cosmetics and the many types of cosmetics circulating in Indonesia need strict supervision by the National Agency of Drug and Food Control (NADFC) to ensure its safety and do not cause irritation and allergies.

Testing irritation and allergies on the skin previously carried out by using rabbits and albino guinea pig, but with the widespread animal welfare issues causing restrictions on the use of experimental animals. So it is necessary to develop the method of skin irritation test in vitro, one of them by using Reconstructed Human Epidermis or RHE (Nelson et al. 1980).

RHE is an artificial epidermis made in such a way that its anatomy and physiology resemble human skin, and can be used for the identification of irritant chemicals, especially for cosmetic products and medical devices (Rehder 2004). Currently RHE is newly produced by European and American countries, whose skin physiology is very different from Indonesian skin physiology, so that RHE production in Indonesia is considered very important, economical in terms of production, and reduce dependence on other countries. Other advantages of in vitro toxicity testing are easy to do, efficient and economical.

\section{Structure of Skin Networks}

The skin is the largest organ of the body and has two major layers, the epidermis and the dermis. Each of these layers has specific functions and structures. The skin tissue structure can be described as Fig. 1.

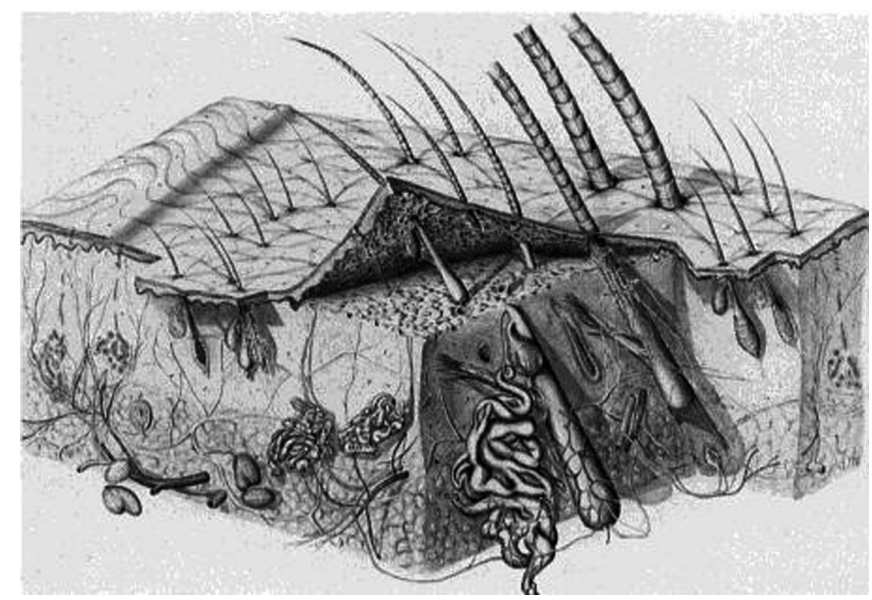

Fig. 1. Diagram of the structure of human skin tissue 
The epidermis occupies the outermost layer, followed by the dermis layer and the hypodermic. The epidermis is an elastic layer and continues to be regenerated, whereas the dermis is the inner layer that includes the sweat glands and produces sweat that travels through the sweat channel to the pores that play a role in temperature regulation. In the layers below the dermis there is a hypodermis composed of fatty tissue or adipose tissue where there are many veins and arteries located between the dermis and hypodermic layers (Montagna 1974).

\section{Epidermis}

The skin epidermis layer consists of the horn layer or corneum layer and the Malpighi layer. The horn or corneum layer is a layer of dead skin, which can be peeled off and replaced by new cells, while the Malpighi layer consists of layers of spinosum and germinativum, each of which has its own function. In addition, the epidermal skin layer contains a melanin pigment that gives color to the skin, therefore it is important to keep the skin from UV radiation that can burn the skin and change the color of the skin becomes darker.

The epidermis is the outermost layer of the human skin and consists of layers such as: melanocytes that produce melanin, langerhans cells which has an important role in skin immunology, and Merkel cells that act as sensory mechanoreceptor. Keratinocytes layer composed of several stratums are the Stratum korneum, Stratum lucidum, Stratum granulosum, Stratum spinosum, Stratum Basal, which is the lowest layer of the epidermis (Schallreuter and dan Wood 1995).

\section{Dermis}

The dermis skin layer consists of blood vessels, hair roots, nerves, sweat glands and oil glands. The function of this dermis skin layer is as a stimulating receptor organ, protective against physical damage, illumination and disease protection, as well as for regulation of body temperature.

The dermis skin layer is at the bottom of the epidermis skin which has two stratum structures, namely the Stratum papilare which contain fibroblasts and stratum reticular which composed of connective tissue (especially type 1 collagen). In addition to these two stratum the dermis also contains some epidermal derivatives namely hair follicles, sweat glands, and sebaceous glands. Below the dermis there is a layer of loose connective tissue called the subcutaneous tissue and contains fat cells. This network is also called superficial fascia or paniculus adiposus. This tissue contains rich bundles of blood vessels and lymph vessels (Montagna 1974). 


\section{Keratinocytes Cells}

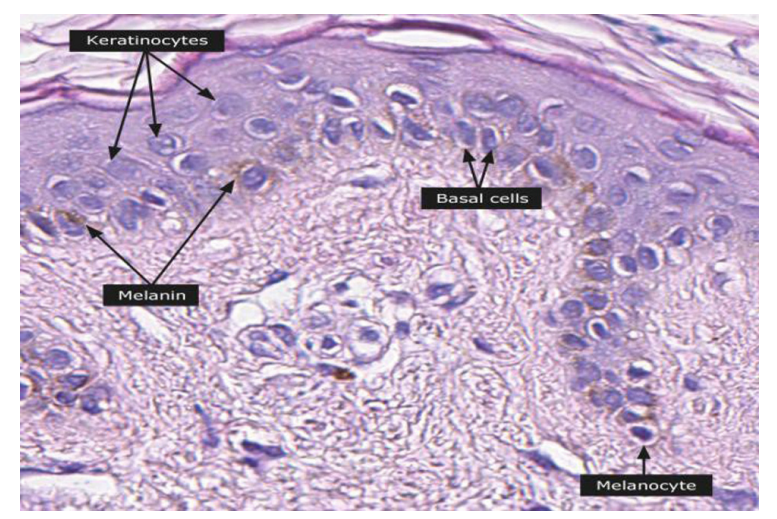

Fig. 2. Skin epidermis with keratinocyte and melanocytes cells

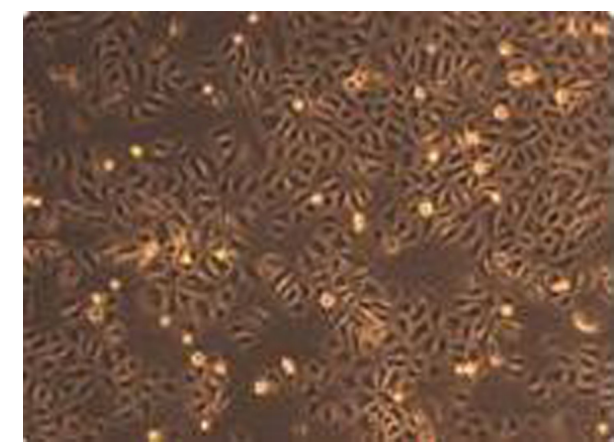

Fig. 3. Keratinocyte cells on day 5 in the media culture

Keratinocytes are a type of cell that dominates $90 \%$ more cells that exist in the outermost layer of the skin (epidermis). Keratinocytes are found in the basal layer (Stratum germinativum) of the skin to be referred to as "basal cells" or "basal keratinocytes". Squamous keratinocytes are found in the oral and esophageal mucosa, as well as the cornea, conjunctiva, and genital epithelium (Morgan 2003) (Figs. 2 and 3).

The main function of keratinocytes is as a barrier to environmental damage such as pathogen bacteria or fungi or viruses, heat, UV radiation and water loss. When pathogen invade the epidermal top layer, keratinocytes may react by producing certain proinflammatory and chemokine mediators such as interleukin 1, interleukin 2, interleukin 6, and TNF- $\alpha$ that attract leukocytes to site of pathogen invasion (Morgan 2003).

A number of structural proteins (filaggrin, keratin), enzymes (proteases), lipids and antimicrobial peptides (defensin) contribute to maintaining important skin barrier functions. Keratinization is part of the formation of a physical barrier (cornification), in 
which keratinocytes produce keratin as an overriding primary protein and eventually undergo programmed cell death. Keratin is what makes up the hair and nails (Morgan 2003).

\section{Melanocytes Cell}

Melanocytes are somatic cells that produce melanin, providing important physiological functions in the epidermal basal layer (stratum basal) of the skin, the middle layer of the eye (uvea), the inner ear, the bone, and the liver. Functions related to human melanin production include photo protection, capture of reactive oxygen, execution of metal ions, and bind to certain drugs and organic chemicals (Tolleson 2005). Melanin is the main pigment responsible for skin color. Melanin is dark and absorbs all UV-B light. This melanin is produced through a process called melanogenesis. This melanogenesis

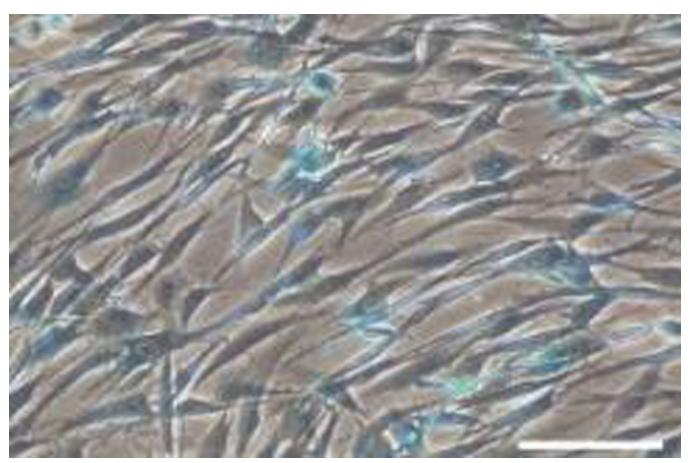

Fig. 4. Melanocyte cell on day 3 in culture medium M 254

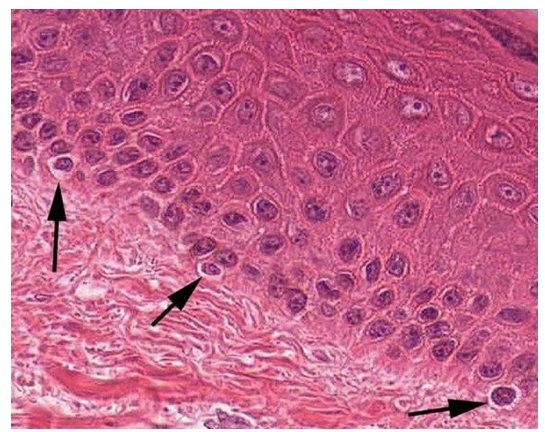

Fig. 5. Melanocyte cells in the epidermal tissue of human skin

causes pigmentation, which is different from the pigmentation derived from the existing melanin oxidation. In general, lighter-skinned people have low-grade basal melanogenesis. Exposure to UV-B radiation causes melanogenesis to increase. The goal of 
melanogenesis is to protect the hypodermis, the lower layers of the skin, from UV-B rays that can damage DNA (Tolleson 2005) (Figs. 4 and 5).

In general, for per square millimeter of skin containing between 1000 and 2000 melanocytes. The difference in skin color between light and dark of a pigmented individual is due to the amount of melanocytes in their skin, but to the extent of melanocyte activity depends greatly on the quantity and relative amount of eumelanin and pheomelanin). This process is under hormonal control, including peptides from Melanocyte-Stimulating Hormones (MSH) and Adrenocorticotropic Hormone (ACTH) resulting from propiomelanocortin precursor.

\section{Fibroblasts Cell}

Fibroblasts are cells that produce collagen and elastin that provide a middle layer of skin structure called the dermis. The dermis is the middle layer of the skin (below the epidermis) containing collagen fibers, elastic fibers, hyaluronic acid, blood vessels and lymphatic vessels, hair follicles, nerves, glands, and many others. This dermis is the "brain" of the skin, also called kutis (Doyle and dan Griffiths 2000).

Fibroblast cells include connective tissue because fibroblast cells have an important role in the formation of connective tissue that is forming fibers (collagen, reticular, and elastin) and producing macromolecules (glycosaminoglycans and proteoglycans) which are the basic components of connective tissue, because the cells are relatively stable and very little of movement.

Elastin is a structural protein that forms thin elastic fibers, similar to collagen. This material is produced by fibroblasts and serves to restore the skin to its position while

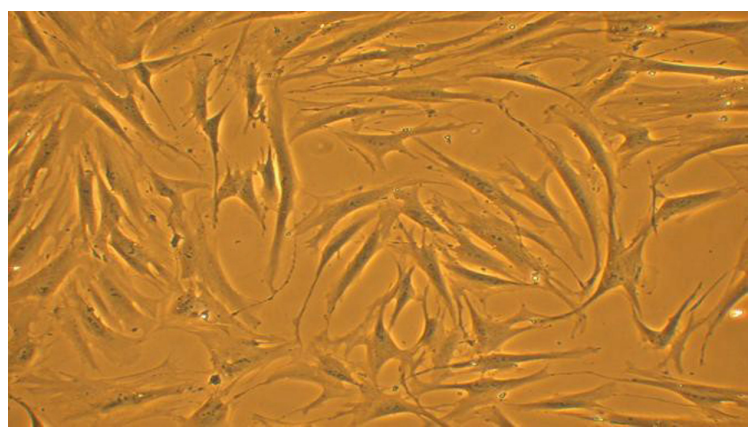

Fig. 6. Fibroblast cells in culture medium containing FBS with 10x magnification

resting. Collagen is a protein that is a major element of connective tissue and bone, and provides strength and endurance to the skin. Aging causes a decrease in collagen production that causes thinning, wrinkled, and sagging skin. Collagen in skin cream is almost useless, but collagen can be stimulated with retinoids, vitamin $\mathrm{C}$ and some other antioxidants, lasers and certain injections (Harlystiarini 2010) (Fig. 6). 


\section{Reconstruct Ed Human Epiderm is (Rhe)}

Reconstructed Human Epidermis (RHE) is defined as human skin tissue obtained from the in vitro process in which human keratinocyte cells are cultured on an inert polycarbonate medium. RHE has a major advantage for growing donor epidermal cells in a serum-free culture environment. After the preparation of the acquired keratinocyte culture has rapidly multiplied, the epidermal cells produced on the inert filter substrate are lifted to the air-fluid interface in the $\mathrm{CO}_{2}$ incubator. A nutrient medium as a food of the basal cells through sub-stratum filters. After 14 days, a stratified epidermis has formed that resembles the human epidermis in vivo. Morphologically, this culture shows stratified epithelial and cornified epidermis by significantly improving barrier function and metabolic activity. Marker differentiation such as keratin suprabasal, integrin b4, integrin a6, fibronectin, involucrin, filaggrin, trichohyalin, type I, III, IV, V and VII collagen, laminin, heparin sulfate and membrane-bound transglutaminase have been found, presented similarly to human epidermis (Rehder 2004).

RHE is formed from a layer of an inert filter substrate containing collagen with fibroblast cells and cultured in an appropriate medium. This layer is a feeder layer that will become the growth medium of keratinocyte cells and melanocytes as the composer of the artificial skin epidermis. RHE can also be composed of keratinocyte cells and melanocytes cultured on biological matrix and the system stored at the air-liquid interface, in a suitable culture medium, to form a human epidermal layer with histologic characteristics of the epidermis in vivo. RHE is considered good when histologic observations indicate reconstructed in vitro epidermis has a similar arrangement of human epidermis in the correct position, with functional keratinocytes and melanocytes similar to in vivo epidermis (Rehder 2004).

The function of RHE is very important as an alternate model of animal experiments for testing the toxicity and effects of topical products since current testing of cosmetic products with experimental animals has been prohibited. In vitro testing using the reconstructed human skin epidermal tissue model (RHE) has been widely used in the cosmetics, pharmaceutical and chemical industries as an alternative to animal testing for various applications including toxicity, efficacy, pharmacology and skin absorption studies. The pharmaceutical and dermocosmetic industries have switched to using the human skin epidermis that was reconstructed into RHE as a model for testing such as: skin penetration rates of various substances, inflammatory reactions, effects of ultraviolet radiation, hormone metabolism and so on. The continuous improvement of this model makes an efficient predictor for research and development of topical toxicity tests (Nelson et al. 1980).

Various Models for epidermal testing have passed scientific validation in ECVAM (European Committee Validation of Alternative Method, a Validation Center for Alternative Methods for European Veterinary Testing) to predict skin irritation and be accepted for regulatory purposes in predicting skin corrosion (OECD 2013) and skin permeation (OECD 2004). But now all models have been properly validated, protected by patents and/or are traded using proprietary tissue culture procedures by some private 
companies, so its availability depends entirely on the strategy of each company (Berking and dan Herlyn 2001).

The epidermis of the skin is an important tissue that produces a strong internal organ body protector, flexible and able to repair itself against environmental influences. Its main function is to protect the body from dehydration, loss of nutrients and unwanted effects due to substances that contact with the skin in various forms depending on its physicochemical properties. The epidermis can contribute to the protection of living organisms that we encounter around us, such as animals, plants and fungi, as well as invisible micro-organisms by the human eye such as bacteria and viruses.

In order to be and remain effective, the epidermal layer should be able to protect the body from the substance most often potentially harmful to physical, chemical or biological. Therefore, most epidermal studies are related to the production, maintenance and repair of the epidermal layer (Poumay and dan Coquette 2007). Since the skin is often exposed to cosmetic products or other ingredients whether intentionally or not, the potential for irritation or corrosion of the skin is large enough that as part of the overall safety assessment process, a product must be carefully evaluated.

\section{Skin Irritation Test}

Skin irritation test can be done both in vivo and in vitro. In vivo testing typically used experimental animals as albino rabbits, while in vitro testing was used as an experimental model of RHE. In vitro testing is a strategy for assessing potential corrosion and irritation. Testing of chemicals for skin irritation after acute exposure is considered to be one way to evaluate the toxicology of simple endpoints. Historically, this process involves only exposure to chemicals in rabbit skin and observing the results (OECD 2010). Over the past decade, various non-animal test methods (in vitro tests) have been developed and validated to predict the potential for skin corrosion and chemical skin irritation (Doucet et al. 2010).

\section{Examination of Histology Network with Hematoxyline - Eosin Determination}

Histology is a branch of biological science that studies the structure of tissues in detail using a microscope on a thinly cut tissue preparation. Histology can also be referred to as a microscopic anatomical science.

Histology is divided into two parts: general histology that includes studies of tissues contained in the body and special histology or organ histology that includes the histological structure of the organs of the body. The tissues of the body are formed by two interacting components of the cell and the extracellular matrix. The extracellular matrix consists of many types of molecules, and most of them are very complex and form complex structures, such as fibers and basement membranes. To analyze the tissue structure that has been cut thinly used the method of haematoxylin-eosin staining (HE), because this coloration can show most of the histology structure. The hematoxillin will 
form the nucleus of the cell (nucleus) and other acidic structures of the cell (such as the RNA-rich portion of the cytoplasm and the cartilage matrix) into blue, while the Eosin is acidic, which will color the cytoplasm and collagen into pink (Dharma 1985).

\section{Immunohistochemical (IHC)}

Immunohistochemistry is a method to detect proteins in cells of a tissue by using the principle of binding between antibodies and antigens in living tissues. Immunohistochemical painting are widely used in the examination of abnormal cells such as cancer cells. Specific molecules will color certain cells such as dividing cells or dead cells that can be distinguished from normal cells. This check requires a network.

of varying amounts and thicknesses depending on the purpose of the inspection. Generally the tissues that come from the body will be cut into very thin pieces by using a tool called vibrating microtome.

Some commonly used immunohistochemical examples include Keratin 10, Carcino Embryonic Antigen (CEA), CD 15, CD 30, Prostate Spesific Antigen (PSA), CD 117, Estrogen and Progesteron, CD 20 and CD 3.

\section{Result and Discussion}

\section{Cell Culture of Keratinocytes, Melanocytes and Fibroblasts}

Keratinocyte cells in culture using Keratinocyte SFM (IX) medium, with addition of human recombinant Epidermal Growth Factor (rEGF) and Bovine Pituitary Extract (BPE). The $7^{\text {th }}$ day keratinocytes and confluent $11^{\text {th }}$ day keratinocytes can be observed with an inverted microscope of $100 \times$ magnification.

Melanocyte cells in culture using 254 Medium plus Human Melanocyte Growth Supplement (HMGS). The 3rd day melanocytes and confluent 6th day of melanocyte can be observed with an inverted microscope of $100 \times$ magnification.

Fibroblast cells in culture using M 106 Medium plus LSGS supplement. The 4th day fibroblast cells and confluent 7 th day of fibroblast can be observed with an inverted microscope of $100 \times$ magnification.

\section{Making of Feeder Layer (Dermis Eqivalen)}

The feeder layer (dermis eqivalen) is made of a mixture of fibroblasts and collagen cells that function as a scaffold in the preparation of RHE. The results show that the eqivalent dermis forms a layer that does not stick to the bottom of the plate, can be observed after incubation for 3 days at $37{ }^{\circ} \mathrm{C}$ using $5 \% \mathrm{CO}_{2}$ incubator. 


\section{Making of RHE Tissue (Reconstructed Human Epidermis)}

The RHE is made of from the eqivalent dermis layer coated with the epidermis layer,

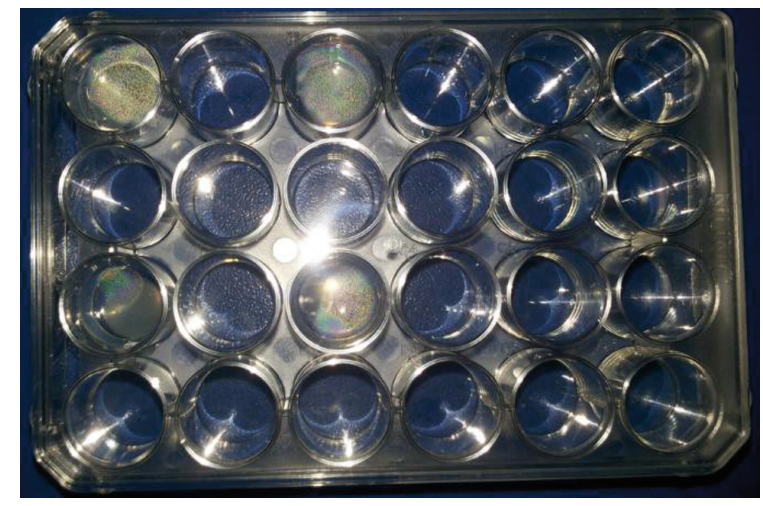

Fig. 7. RHE tissue consisting of an equivalent dermis and epidermal layer

where the epidermis layer is on the seedling above the sterile insert comprising a mixture of keratinocyte cells and melanocyte cells in ratio (40:1), then cultured on an epidermal medium (IMDM) containing vitamin C $37.5 \mu \mathrm{g} / \mathrm{mL}$. The purpose of adding vitamin $C$ to the culture medium is as an antioxidant to strengthen the epidermal membrane layer so that the layer is not easily separated. The results of making RHE network can be seen in Fig. 7.

\section{Measuring of Cell Life Using MTT Method}

Measurements of cell life from RHE tissue were used MTT method, wherein keratinocyte and melanocyte cell life was obtained by calculation using formula:

$$
\% \text { celllife }=\frac{\text { treatmentabsorption }- \text { blankoabsorption } \times 100 \% \text { Blankoabsorption }}{\text { Blankoabsorption }}
$$

Measurements of cell life from the epidermal layer were performed on day 1 (considered as base line), day 3, day 7, day 11 and day 14 . The increase in the number of living cells occurred only until the 7 th day, then there was a decrease on the 11th day. This may be due to the fact that the number of keratinocyte and melanocyte cells grown too much, so that the availability of culture media on the propylene ring is insufficient, eventually the growth of cells in the tissue becomes blocked and some die.

Epidermis layer production is repeated by decreasing the number of planting cells to one fifth of the number of cells grown in the first experiment. So the number of cells changed from $50 \times 104$ cell $/ \mathrm{mL}$ to $10 \times 104$ cell $/ \mathrm{mL}$ for keratinocyte cells and $1,25 \times$ 104 cell $/ \mathrm{mL}$ to $0,25 \times 104$ cell $/ \mathrm{mL}$ for melanocyte cells. 


\section{Making Histology Preparations of Reconstructed Human Epiderm Using Staining of Hematoxylin-Eosin (HE)}

Hematoxylin and Eosin are coloring methods that are widely used in the staining of histological tissues. Hematoxylin memulas the nucleus and other acidic structures of

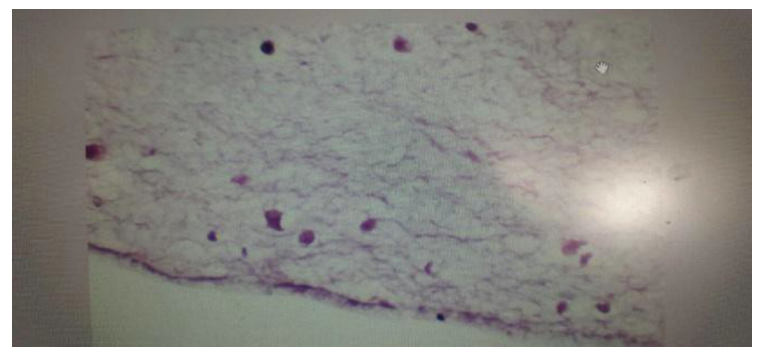

Fig. 8. The results of skin tissue reconstruction on the 11th day

the cell (such as the RNA-rich cytoplasm section and cartilage matrix) to blue/purple. Haematoxylin will color the nucleus while eosin will color the cytoplasm. Eosin is acidic, which will color the cytoplasm and collagen into pink.

The result of preparation of histology and staining using HE can be seen in Fig. 8 here is the presence of cell nucleus (purple blue color) but not yet showing the formation of tissue component (not yet showing any protein expressed in skin tissue), so immunohistochemistry is needed antibody (marker) that is specific to skin tissue.

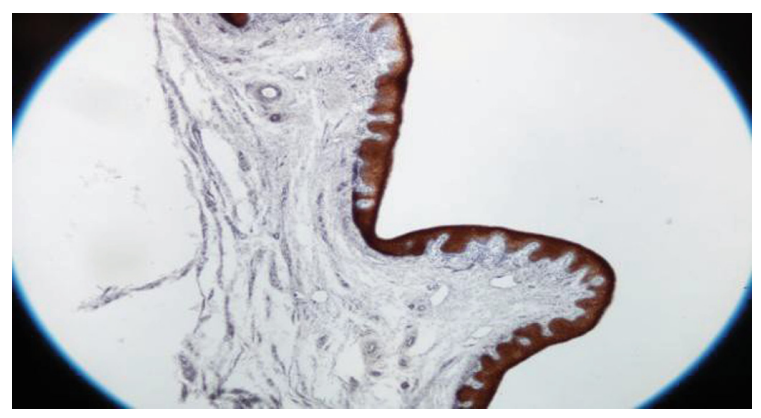

Fig. 9. IHC examination of normal skin tissue 


\section{Immunohistochemical (IHC) Examination of RHE Skin Tissue Using Cytokeratin 10 Antibody Marker}

Immunohistochemical (IHC) is a technique for detecting a specific protein (antigen) in tissues or cells using specific antibodies (markers). The purpose of this IHC examination is to validate the reconstructed skin tissue, using antibodies specific to the epidermis. The results of immunohistochemical examination of RHE skin tissue can be seen in Fig. 9.

The results of IHC examination of reconstructed skin tissue using cytokeratin 10 antibody marker have not shown any integration (bonding) between the epidermal layer with the marker, this is probably because the proteins formed are incompatible with antibody cytokeratin 10 . Other possibilities is not yet formed protein so physiological function of the epidermis has not existed, therefore RHE skin tissue cannot be used as skin irritation test model in vitro.

\section{Conclusion}

Keratinocyte, melanocytes and fibroblasts cells can grow well on suitable medium with the addition of suitable growth medium that is keratinocyte cells grown on Keratinocyte SFM (IX) culture medium with Human recombinant supplement (rEGF); melanocyte cells grown on Melanocyte 254 culture medium with HMGS.

supplementation; and fibroblast cells grown on Fibroblast M 106 culture medium with LSGS supplementation. The percentage of epidermal cell life grew well in the planting of $10 \times 104$ cells $/ \mathrm{mL}$ keratinocytes and $0.25 \times 104$ cells $/ \mathrm{mL}$ of melanocyte cells and survived until the 11th day with live cell percentage of $93.45 \%$. The preparation of histology preparations with HE staining shows the presence of cell life in RHE tissue. IHC examination using cytokeratin 10 antibody marker not shows any physiological function of the epidermis tissue.

\section{References}

Berking C, dan Herlyn M (2001) Human skin reconstruct models: a new applicationfor studies of melanocyte and melanoma. Biology Histol Histopathol 16:669-674

Dharma A (1985) Basic Histology

Doucet $O$ et al (2010) A catch-up validation study on reconstructed human epidermis (SkinEthicTM RHE) for full replacement of the Draize skin irritation test, Toxicology in Vitro, 257-266

Doyle A, dan Griffiths JB (2000) Cell and tissue culture for medical research. John Willey \& Sons Ltd, England

Harlystiarini (2010) In Vitro Fibroblast in culture. Bogor Agriculture University

Montagna W (1974) The structure and function of skin. Academy Press, New York, London 
Morgan GA (2003) Experimental model of cultured keratinocytes. Acta Cir Bras [serial online], vol 18 Special Edition

Nelson PH et al (1980) Optimized condition for the growth of human epidermis cell in culture. J Investig Dermatol 75:176-182

OECD (2004) Guideline For The Testing Of Chemicals. In Vitro Skin Irritation Reconstructed Human Epidermis Test Method, Test No, p 428

OECD (2010) Guideline for the testing of chemicals. In Vitro skin irritation reconstructed human epidermis test method, Test No. 439

OECD (2013) Guideline for the testing of chemicals. In Vitro skin corrosion: reconstructed human epidermis (RHE) Test method Test No. 431

Poumay Y, dan Coquette A (2007) Modelling the human epidermis in vitro: tools for basic and applied research. Arch Dermatol Res 298:361-369

Rehder J et al (2004) Model of human epidermis reconstructed in vitro with keratinocytes and melanocytes on dead de-epidermized human dermis. Sao Paulo Med J 122(1):22-25

Schallreuter KU, dan Wood JM (1995) The human epidermis. Proc Nutrition Soc 54:191-195

Tolleson WH (2005) Human melanocyte biology. Toxicol Pathol J Environ Sci Health 23:105161

Open Access This chapter is licensed under the terms of the Creative Commons Attribution 4.0 International License (http://creativecommons.org/licenses/by/4.0/), which permits use, sharing, adaptation, distribution and reproduction in any medium or format, as long as you give appropriate credit to the original author(s) and the source, provide a link to the Creative Commons licence and indicate if changes were made.

The images or other third party material in this chapter are included in the chapter's Creative Commons licence, unless indicated otherwise in a credit line to the material. If material is not included in the chapter's Creative Commons licence and your intended use is not permitted by statutory regulation or exceeds the permitted use, you will need to obtain permission directly from the copyright holder.

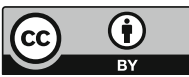

